\title{
Model Reduction by Moment Matching for Linear Singular Systems
}

\author{
Giordano Scarciotti
}

\begin{abstract}
The paper presents a moment matching approach to the model reduction problem for singular systems. Combining the interpolation-based and the steady-state-based description of moment, a partitioned formulation of the Krylov projector is obtained. Several implications of this result are investigated and different families of reduced order models are proposed. The possibility to maintain structural properties of the fast subsystem is studied. Two examples illustrate the results of the paper.
\end{abstract}

\section{INTRODUCTION}

Singular systems, also known as generalized state space systems, descriptor systems or differential-algebraic equations, have been largely investigated because of their capacity of modeling a large variety of physical behaviors such as power systems, electrical networks, chemical processes, biological systems and social economic systems, see [1], [2]. The important characteristic of this class of systems is that they combine dynamic (differential) and static (algebraic) equations. They are often the result of the interconnection of several subsystems and the overall singular system has often large dimensionality. This justifies the interest in the model reduction problem for this class of systems. A first result of model reduction for singular systems has been proposed in [3] in which the chained aggregation method has been used. As pointed out in [4] this method is computationally intensive and another approach based on balanced realization has been proposed. However, the reduced order model obtained therein is a non-singular one and the technique is not able to maintain the impulsive characteristic of singular systems. In [5] a singular reduced order model based on the Nehari's approximation algorithm [6] has been presented. Other results of model reduction via covariance approximation [7] and using singular value decomposition [8] have also been proposed.

In this paper we follow the moment matching approach, see e.g. [9], [10], [11], [12], [13], [14] and [15]. In particular we extend the model reduction method developed in [16] to singular systems. This method has the ability to preserve in a direct way some properties of the original system, overcoming some of the drawbacks of the moment matching methods. Moreover, it can be extended to nonlinear systems, see e.g. [17], [18] and [19]. Combining the interpolationbased and the steady-state-based description of moment (see [20] and [21] for a detailed discussion of the two descriptions) a partitioned projector $\Pi$ is computed. In the projector, the contribution of the fast subsystem is separated by the

G. Scarciotti is with the Dept. of Electrical and Electronic Engineering, Imperial College London, London, SW7 2AZ, UK, E-mail: gs3610@ic.ac.uk. contribution of the slow subsystem. This property, which has several consequences, is the main contribution of this paper. In fact, the information on the fast subsystem is clearly encoded in the projector and it is not lost by the moment matching technique. Moreover, the output of reduced order models based on this projector approximates the output of the system only when consistent initial conditions are taken in account. In addition, the partition of the projector allows to define several families of reduced order models which have great flexibility in maintaining independently specific properties of the slow and fast subsystems. Thus, purely fast, purely slow and a "simple" family of reduced order models are proposed and the possibility of matching with impulsive controllability constraints is discussed.

The rest of the paper is organized as follows. In Section II we recall some basic results for singular systems which are instrumental to develop the remaining of the paper. In Section III the interpolation-based description of moment is extended to singular systems. Then, combining it with the steady-state-based description of moment, a new formulation of the projector $\Pi$ is given. In Section IV several families of reduced order models are given and the possibility to retain, reduce or eliminate the fast subsystem in the reduced order model is investigated. Moreover, a result to enforce impulsive controllability on the reduced order model is proposed. In Section V two numerical examples are used to show the application of the results of the paper. Finally Section VI contains some concluding remarks and future directions of research.

Notation. We use standard notation. $\mathbb{C}_{<0}$ denotes the set of complex numbers with negative real part, whereas $\emptyset$ indicates the empty set. The symbol $I$ denotes the identity matrix and $\sigma(A)$ denotes the spectrum of the matrix $A \in \mathbb{R}^{n \times n}$. The symbol $\otimes$ indicates the Kronecker product. The vectorization of a matrix $A \in \mathbb{R}^{n \times m}$, denoted by $\operatorname{vec}(A)$, is the $n m \times 1$ vector obtained by stacking the columns of the matrix $A$ one on top of the other, namely $\operatorname{vec}(A)=\left[a_{1}^{\top}, a_{2}^{\top}, \ldots, a_{m}^{\top}\right]^{\top}$, where $a_{i} \in \mathbb{R}^{n}$ are the columns of $A$ and the superscript $\top$ denotes the transpose.

\section{Preliminaries on Singular Systems}

In this section some basic results for singular systems are recalled. Consider a linear, single-input, single-output, continuous-time, singular system described by the equations ${ }^{1}$

$$
E \dot{x}=A x+B u, \quad y=C x,
$$

\footnotetext{
${ }^{1}$ The results can be extended to multi-input, multi-output (MIMO) systems straightforwardly. The problem in the MIMO case is called tangential interpolation and is discussed, in the present context, in [22], see also [23].
} 
with $x(t) \in \mathbb{R}^{n}, u(t) \in \mathbb{R}, y(t) \in \mathbb{R}, E \in \mathbb{R}^{n \times n}, A \in \mathbb{R}^{n \times n}$, $B \in \mathbb{R}^{n \times 1}$ and $C \in \mathbb{R}^{1 \times n}$. Assume that $u(t)$ is piecewise continuously differentiable.

Definition 1: [1] Let $\mathcal{E} \subset \mathbb{C}$ be the set of complex numbers $s$ such that $\operatorname{det}(s E-A) \neq 0$. For any two matrices $E$ and $A$, the pencil $(E, A)$ is called regular if $\mathcal{E} \not \equiv \emptyset$.

Lemma 1: [1] The pencil $(E, A)$ is regular if and only if there exist two nonsingular matrices $Q$ and $P$ such that

$$
Q E P=\operatorname{diag}(I, N), \quad Q A P=\operatorname{diag}\left(A_{1}, I\right),
$$

where $N \in \mathbb{R}^{n_{2} \times n_{2}}$ is nilpotent with degree ${ }^{2} h$ and $A_{1} \in$ $\mathbb{R}^{n_{1} \times n_{1}}$ is full rank, with $n_{1}+n_{2}=n$.

Assume system (1) is regular, then $Q$ and $P$ can be selected such that system (1) can be written in the so-called first equivalent form, namely

slow subsystem:

fast subsystem:

$$
\begin{aligned}
\dot{x}_{1} & =A_{1} x_{1}+B_{1} u, \\
y_{1} & =C_{1} x_{1}, \\
y & =y_{1}+y_{2},
\end{aligned}
$$$$
N \dot{x}_{2}=x_{2}+B_{2} u,
$$$$
y_{2}=C_{2} x_{2} \text {, }
$$

with the coordinate transformation $\left[\begin{array}{ll}x_{1}^{\top} & x_{2}^{\top}\end{array}\right]^{\top}=P^{-1} x$, where $x_{1} \in \mathbb{R}^{n_{1} \times n_{1}}, x_{2} \in \mathbb{R}^{n_{2} \times n_{2}}$, and

$$
\begin{array}{ll}
Q E P=\operatorname{diag}(I, N), & Q B=\left[\begin{array}{ll}
B_{1}^{\top} & B_{2}^{\top}
\end{array}\right]^{\top}, \\
Q A P=\operatorname{diag}\left(A_{1}, I\right), & C P=\left[\begin{array}{ll}
C_{1} & C_{2}
\end{array}\right] .
\end{array}
$$

The state response of system (1) is given by

$$
\begin{aligned}
x(t)=P\left[\begin{array}{l}
I \\
0
\end{array}\right] & \left(e^{A_{1} t} x_{1}(0)+\int_{0}^{t} e^{A_{1}(t-\tau)} B_{1} u(\tau) d \tau\right) \\
& -P\left[\begin{array}{l}
0 \\
I
\end{array}\right] \sum_{i=0}^{h-1} N^{i} B_{2} \frac{d^{i}}{d t^{i}} u(t) .
\end{aligned}
$$

Differently from standard linear systems, singular systems have a unique solution only if $x(0)=$ $P\left[\begin{array}{ll}x_{1}^{\top}(0) & x_{2}^{\top}(0)\end{array}\right]^{\top}$ is a consistent initial condition, namely it is such that

$$
x(0)=P\left[\begin{array}{l}
I \\
0
\end{array}\right] x_{1}(0)-\left.P\left[\begin{array}{l}
0 \\
I
\end{array}\right] \sum_{i=0}^{h-1} N^{i} B_{2} \frac{d^{i}}{d t^{i}} u(t)\right|_{0},
$$

where $x_{1}(0)$ can be freely selected.

\section{DEFINITION OF MOMENT}

In this section we establish a one-to-one relation between the moments of system (1), the unique solution of a generalized Sylvester equation and the steady-state response of the output of a particular interconnected system. Let

$$
\begin{aligned}
W(s) & =C(s E-A)^{-1} B \\
& =C_{1}\left(s I-A_{1}\right)^{-1} B_{2}+C_{2}(s N-I)^{-1} B_{2},
\end{aligned}
$$

be the transfer function associated to system (1) and assume that the system is minimal, i.e., as for non-singular systems (see [1, Theorem 2-6.3]), controllable and observable.

\footnotetext{
${ }^{2}$ A nilpotent matrix is a square matrix $N$ such that $N^{k}=0$ for some positive integer $k$. The smallest such $k$ is called the degree of $N$.
}

\section{A. Interpolation-based description of moment}

We begin extending the classical interpolation-based description of moment. This, in conjunction with the steadystate-based description of moment, allows to obtain a special structure for the projector to be used in the model reduction process.

Definition 2: Let $s_{i} \in \mathcal{E}$. The 0-moment of system (1) at $s_{i}$ is the complex number $\eta_{0}\left(s_{i}\right)=C\left(s_{i} E-A\right)^{-1} B$. The $k$-moment of system (1) at $s_{i}$ is the complex number

$$
\eta_{k}\left(s_{i}\right)=\frac{(-1)^{k}}{k !}\left[\frac{d^{k}}{d s^{k}} W(s)\right]_{s=s_{i}},
$$

with $k \geq 1$ integer.

Note that each moment is the sum of two contributions, one depending on the slow subsystem and one depending on the fast subsystem, i.e.

$$
\begin{aligned}
\eta_{k}\left(s_{i}\right)=(-1)^{k} C_{1}( & \left.s_{i} I-A_{1}\right)^{-(k+1)} B_{1} \\
+ & (-1)^{k} C_{2} N^{k}\left(s_{i} N-I\right)^{-(k+1)} B_{2},
\end{aligned}
$$

and if $k \geq h$,

$$
\eta_{k}\left(s_{i}\right)=(-1)^{k} C_{1}\left(s_{i} I-A_{1}\right)^{-(k+1)} B_{1} .
$$

This definition of moment is justified by the fact that the $k$-moment of a linear system at $s_{i}$ is defined as the $k$-th coefficient of the Laurent series expansion of the transfer function $W(s)$ at $s_{i} \in \mathbb{C}$ (see [9, Chapter 11]), provided it exists. The next result, which is a direct extension of [16] (see also [24] and [14]), gives a relation between the moments and the solution of a generalized Sylvester equation.

Lemma 2: Let $s_{i} \in \mathcal{E}$. Consider system (1), then

$$
\left[\begin{array}{lll}
\eta_{0}\left(s_{i}\right) & \ldots & \eta_{k}\left(s_{i}\right)
\end{array}\right]=C \widetilde{\Pi} \Psi_{k}
$$

where $\Psi_{k}=\operatorname{diag}\left(1,-1,1, \ldots,(-1)^{k}\right) \in \mathbb{R}^{(k+1) \times(k+1)}$ and $\widetilde{\Pi}$ is the unique solution of the generalized Sylvester equation

$$
A \widetilde{\Pi}+B L_{k}=E \widetilde{\Pi} \Sigma_{k},
$$

with $L_{k}=\left[\begin{array}{llll}1 & 0 & \ldots & 0\end{array}\right] \in \mathbb{R}^{(k+1)}$ and

$$
\Sigma_{k}=\left[\begin{array}{ccccc}
s_{i} & 1 & 0 & \ldots & 0 \\
0 & s_{i} & 1 & \ldots & 0 \\
\vdots & \vdots & \ddots & \ddots & \vdots \\
0 & \cdots & 0 & s_{i} & 1 \\
0 & \cdots & \cdots & 0 & s_{i}
\end{array}\right] \in \mathbb{R}^{(k+1) \times(k+1)}
$$

Proof: Let $\widetilde{\Pi}=\left[\begin{array}{llll}\widetilde{\Pi}_{0} & \widetilde{\Pi}_{1} & \ldots & \widetilde{\Pi}_{k}\end{array}\right]$ and note that (5) can be rewritten as

$$
\begin{array}{ll}
A \widetilde{\Pi}_{0}+B & =E \widetilde{\Pi}_{0} s_{i} \\
A \widetilde{\Pi}_{1} & =E \widetilde{\Pi}_{1} s_{i}+E \widetilde{\Pi}_{0} \\
& \vdots \\
A \widetilde{\Pi}_{k} & =E \widetilde{\Pi}_{k} s_{i}+E \widetilde{\Pi}_{k-1} .
\end{array}
$$


As a result

$\widetilde{\Pi}_{0}=(s E-A)^{-1} B$,

$\widetilde{\Pi}_{1}=-(s E-A)^{-1} E(s E-A)^{-1} B=\left[\frac{d}{d s}(s E-A)^{-1} B\right]_{s_{i}}$,

$\widetilde{\Pi}_{k}=\frac{1}{k !}\left[\frac{d^{k}}{d s^{k}}(s E-A)^{-1} B\right]_{s_{i}}$,

which proves the claim.

Exploiting this lemma, the following result holds.

Theorem 1: Consider system (1) and suppose that for a set of numbers $s_{i}$, with $i=1, \ldots, \eta, s_{i} \in \mathcal{E}$. Then there exists a one-to-one relation between the moments $\eta_{0}\left(s_{1}\right)$, $\ldots, \eta_{k_{1}-1}\left(s_{1}\right), \ldots, \eta_{0}\left(s_{\eta}\right), \ldots, \eta_{k_{\eta}-1}\left(s_{\eta}\right)$ and the matrix $C \Pi$, where $\Pi$ is the unique solution of the generalized Sylvester equation

$$
A \Pi+B L=E \Pi S,
$$

with $S \in \mathbb{R}^{\nu \times \nu}$ any non-derogatory matrix with characteristic polynomial

$$
p(s)=\prod_{i=1}^{\eta}\left(s-s_{i}\right)^{k_{i}}
$$

where $\nu=\sum_{i=1}^{\eta} k_{i}$ and $L$ such that the pair $(L, S)$ is observable.

Proof: We begin proving that if $\sigma(S) \subset \mathcal{E}$ than equation (6) has a unique solution. Using the vectorization transformation and the Kronecker product properties we obtain the solution

$$
\operatorname{vec}(\Pi)=-\left(I \otimes A-S^{\top} \otimes E\right)^{-1} \operatorname{vec}(B L),
$$

which is unique if and only if

$$
\operatorname{det}\left(I \otimes A-S^{\top} \otimes E\right) \neq 0 .
$$

Let $R$ be an invertible matrix such that $R^{-1} S R=J_{S}$, where $J_{S}$ is the complex Jordan form of $S$. Multiplying on the left by $(R \otimes I)$ and on the right by $\left(R^{-1} \otimes I\right)$ we obtain the equivalence

$$
\begin{aligned}
& \operatorname{det}\left(I \otimes A-S^{\top} \otimes E\right)= \\
& =\operatorname{det}\left((R \otimes I)\left(I \otimes A-S^{\top} \otimes E\right)\left(R^{-1} \otimes I\right)\right)= \\
& \quad=\operatorname{det}\left(I \otimes A-J_{S}^{\top} \otimes E\right) .
\end{aligned}
$$

Then (8) holds if and only if

$$
\prod_{i=1}^{\eta} \operatorname{det}\left(A-s_{i} E\right) \neq 0 \text {. }
$$

For the remaining of the theorem, it is sufficient to prove the claim for $\eta=1$. By observability of the pair $(L, S)$ there is a unique invertible matrix $T$ such that $S=T^{-1} \Sigma_{k} T$ and $L=L_{k} T$. Then equation (6) becomes

$$
A \Pi T^{-1} T-E \Pi T^{-1} \Sigma_{k} T=-B L_{k} T .
$$

The claim follows defining $\widetilde{\Pi}=\Pi T^{-1}$, multiplying on the right by $T^{-1}$ and recalling that the moments are coordinates invariant.

\section{B. Steady-state-based description of moment}

In this section we present the steady-state-based description of moment for linear singular systems. This is instrumental to derive the main technical result of the paper, namely a partition of the projector $\Pi$ in slow and fast parts.

Theorem 2: Let $S \in \mathbb{R}^{\nu \times \nu}$ be any non-derogatory matrix with characteristic polynomial (7). Consider system (1) and suppose that $\sigma(S) \subset \mathcal{E}$ and $\sigma(A) \subset \mathbb{C}_{<0}$. Consider the interconnection of system (1) with the system

$$
\dot{\omega}=S \omega, \quad u=L \omega,
$$

with $L$ and $\omega(0)$ such that the triple $(L, S, \omega(0))$ is minimal. Then there exists a one-to-one ${ }^{3}$ relation between the moments $\eta_{0}\left(s_{1}\right), \ldots, \eta_{k_{1}-1}\left(s_{1}\right), \ldots, \eta_{0}\left(s_{\eta}\right), \ldots, \eta_{k_{\eta}-1}\left(s_{\eta}\right)$ and the steady-state response of the output of such interconnected system.

Proof: Consider the interconnection of system (1) with system (9). By the assumptions on the pencil $(E, A)$ and $\sigma(S)$, the interconnected system has a well-defined invariant manifold given by $\mathcal{M}=\left\{(x, \omega) \in \mathbb{R}^{n+\nu}: x=\Pi \omega\right\}$, with $\Pi$ the unique solution of the generalized Sylvester equation (6). We prove now that $\mathcal{M}$ is attractive. Consider the equation

$$
\overbrace{E x-E \Pi \omega}^{i}=A x+B L \omega-E \Pi S \omega,
$$

in which substituting (6), yields

$$
\overbrace{E x-E \Pi \omega}^{-i}=A(x-\Pi \omega) \text {. }
$$

Let $z=x-\Pi \omega$, then

$$
E \dot{z}=A z .
$$

The state response of this last equation is

$$
z(t)=P\left[\begin{array}{l}
I \\
0
\end{array}\right] e^{A_{1} t} z(0) .
$$

Thus, the output response of the interconnected system is

$$
y(t)=C \Pi \omega(t)+C P\left[\begin{array}{l}
I \\
0
\end{array}\right] e^{A_{1} t}\left(x_{1}(0)-\Pi \omega(0)\right) .
$$

The claim follows observing that

$$
C P\left[\begin{array}{l}
I \\
0
\end{array}\right] e^{A_{1} t}\left(x_{1}(0)-\Pi \omega(0)\right),
$$

describes the transient response which vanishes exponentially.

We are ready now to give the main result of the paper.

Theorem 3: Let $S \in \mathbb{R}^{\nu \times \nu}$ be any non-derogatory matrix with characteristic polynomial (7). Consider system (2) and

\footnotetext{
${ }^{3}$ By one-to-one relation we mean that the moments are uniquely determined by the steady-state response of $y(t)$ and vice versa.
} 
suppose that $\sigma(S) \cap \sigma\left(A_{1}\right)=\emptyset$. Then there exists a one-toone relation between the moments $\eta_{0}\left(s_{1}\right), \ldots, \eta_{k_{1}-1}\left(s_{1}\right)$, $\ldots, \eta_{0}\left(s_{\eta}\right), \ldots, \eta_{k_{\eta}-1}\left(s_{\eta}\right)$ and the matrix $C \widehat{\Pi}$, where $\widehat{\Pi}=P^{-1} \Pi=\left[\begin{array}{ll}\Pi_{1}^{\top} & \Pi_{2}^{\top}\end{array}\right]^{\top}$ is the unique solution of the equations

$$
\begin{aligned}
A_{1} \Pi_{1}-\Pi_{1} S & =-B_{1} L \\
\Pi_{2} & =-\sum_{i=0}^{h-1} N^{i} B_{2} L S^{i} .
\end{aligned}
$$

Proof: In steady-state, using the change of coordinates

$$
\left[\begin{array}{l}
x_{1 s s} \\
x_{2 s s}
\end{array}\right]=P^{-1} x_{s s}=P^{-1} \Pi \omega=\left[\begin{array}{l}
\Pi_{1} \\
\Pi_{2}
\end{array}\right] \omega,
$$

in (2), yields

$$
\begin{aligned}
\Pi_{1} S \omega & =A_{1} \Pi_{1} \omega+B_{1} L \omega, \\
N \Pi_{2} S \omega & =\Pi_{2} \omega+B_{2} L \omega,
\end{aligned}
$$

from which $\omega$ can be eliminated because the two equations hold for any $\omega$. We readily obtain the first of equations (10). Applying the vectorization transformation and the Kronecker product properties to the second equation we see that $\Pi_{2}$ is unique if and only if

$$
\operatorname{det}\left(I-s_{i} N\right) \neq 0
$$

for any $s_{i} \in \sigma(S)$. This holds by definition for any $s_{i} \in \mathbb{C}$ since $N$ is a nilpotent matrix. Then, $\Pi$ is unique if and only if $\sigma(S) \cap \sigma\left(A_{1}\right)=\emptyset$. To obtain the explicit expression of $\Pi_{2}$, we substitute $x_{2 s s}=\Pi_{2} \omega$ in the last $n_{2}$ equations in (3) obtaining

$$
\Pi_{2} \omega=-\sum_{i=0}^{h-1} N^{i} B_{2} L \frac{d^{i}}{d t^{i}} \omega .
$$

Observing that $\frac{d^{i}}{d t^{i}} \omega=S^{i} \omega$, yields

$$
\Pi_{2}=-\sum_{i=0}^{h-1} N^{i} B_{2} L S^{i}
$$

since the equation holds for any $\omega$.

This result has multiple implications. The contribution to the moment of the fast subsystem is separated in the projector $\widehat{\Pi}$ from the contribution of the slow subsystem. The condition for uniqueness of the projector is simplified and it depends only on the eigenvalues of the slow subsystem. This is actually expected since in the first equivalent form the $k$ th moment of the system can be written as (4), in which $s_{i} N-I$ is full-rank for any $s_{i}$.

It is clear from the form of $\widehat{\Pi}$ that the information on the fast subsystem is encoded in the projector and it is not lost by the moment matching technique. Thus, the output of reduced order models based on this projector approximates the output of the system only when consistent initial conditions are taken in account, i.e. when the solution of the system to be reduced exists. Moreover, this formulation gives high flexibility in maintaining the properties of the fast and slow subsystems. Depending on the particular application the fast subsystem can be preserved, reduced or eliminated independently of the reduction of the slow subsystem. This possibility is analyzed in detail in the next section.

\section{REDUCED ORDER MODELS}

In this section several families of reduced order models achieving moment matching are presented. The possibility of obtaining purely fast or purely slow singular systems is investigated and a "simple" family of singular systems is given. In addition, the solution to the problem of matching with impulsive controllability is given.

Definition 3: Consider system (1) and let $S \in \mathbb{R}^{\nu \times \nu}$ be any non-derogatory matrix with characteristic polynomial (7). Assume $\sigma(S) \cap \sigma\left(A_{1}\right)=\emptyset$ and let $L$ be such that the pair $(L, S)$ is observable. Then the system

$$
\begin{aligned}
\dot{\xi}_{1} & =F_{1} \xi_{1}+G_{1} u, & M \dot{\xi}_{2}=\xi_{2}+G_{2} u, \\
\psi_{1} & =H_{1} \xi_{1}, & \psi_{2}=H_{2} \xi_{2}, \\
\psi & =\psi_{1}+\psi_{2}, &
\end{aligned}
$$

with $\xi_{j}(t) \in \mathbb{R}^{\nu}$, for $j=1,2, \psi_{j}(t) \in \mathbb{R}$, for $j=1,2$, $F_{1} \in \mathbb{R}^{\nu \times \nu}$ full-rank, $M \in \mathbb{R}^{\nu \times \nu}$ nilpotent with degree $\hbar$, $G_{j} \in \mathbb{R}^{\nu \times 1}$, for $j=1,2$, and $H_{j} \in \mathbb{R}^{1 \times \nu}$, for $j=1,2$, is a model of system (1) at $S$, if there exists a unique solution $\left[\begin{array}{cc}\Gamma_{1}^{\top} & \Gamma_{2}^{\top}\end{array}\right]^{\top}$ of the equations

$$
\begin{aligned}
F_{1} \Gamma_{1}-\Gamma_{1} S & =-G_{1} L \\
\Gamma_{2} & =-\sum_{i=0}^{\hbar-1} M^{i} G_{2} L S^{i},
\end{aligned}
$$

such that

$$
\begin{aligned}
& C_{1} \Pi_{1}=H_{1} \Gamma_{1}, \\
& C_{2} \Pi_{2}=H_{2} \Gamma_{2},
\end{aligned}
$$

where $\left[\begin{array}{ll}\Pi_{1}^{\top} & \Pi_{2}^{\top}\end{array}\right]^{\top}$ is the unique solution of (10). System (11) is a reduced order model of system (1) at $S$ if $2 \nu<n$.

We analyze now some special families of reduced order models which achieve moment matching.

1) Non-singular reduced order model: using the results of the paper, it is easy to approximate a singular system with a non-singular system. This was the first approach attempted, see [4]. The same authors and following researchers, e.g. see [5], deemed the method inaccurate, since the impulsive characteristic of the singular system is lost. However, in particular settings and as a first approximation it can be useful to approximate a singular system with a non-singular one. This can be done easily in the presented framework. In fact, the system

$$
\dot{\xi}=F \xi+G u, \quad \psi=H \xi,
$$

is a model of system (1) at $S$, if there exists a unique solution $\Gamma$ of the equation

$$
F \Gamma-\Gamma S=-G L
$$


such that

$$
C \Pi=H \Gamma,
$$

where $\Pi$ is the unique solution of (6). Note that, the reduced order model (14) has dimension $\nu$, whereas the reduced order model (11) has dimension $2 \nu$.

2) Fast reduced order model: in the same way, a completely fast reduced order model which approximates the singular system can be obtained in the presented framework. The system

$$
M \dot{\xi}=\xi+G u, \quad \psi=H \xi,
$$

is a model of system (1) at $S$, if

$$
H=-C \Pi\left(\sum_{i=0}^{\hbar-1} M^{i} G L S^{i}\right)^{-1},
$$

where $\Pi$ is the unique solution of (6), is uniquely determined. The reduced order model (17) has dimension $\nu$.

3) The identity family of singular reduced order models: as already shown in [16], see also [18], a simple family of reduced order models is identified by the choice $\Gamma=I$. In the present setting it seems natural to select $\Gamma_{1}=I$ keeping $M$ and $G_{2}$ free. Then, the system

$$
\begin{aligned}
\dot{\xi}_{1} & =\left(S-G_{1} L\right) \xi_{1}+G_{1} u \\
M \dot{\xi}_{2} & =\xi_{2}+G_{2} u \\
\psi_{1} & =C_{1} \Pi_{1} \xi_{1} \\
\psi_{2} & =C_{2} \sum_{i=0}^{h-1} N^{i} B_{2} L S^{i}\left(\sum_{i=0}^{\hbar-1} M^{i} G_{2} L S^{i}\right)^{-1} \xi_{2}, \\
\psi & =\psi_{1}+\psi_{2},
\end{aligned}
$$

where $\left[\begin{array}{ll}\Pi_{1}^{\top} & \Pi_{2}^{\top}\end{array}\right]^{\top}$ is the unique solution of (10), is a model of system (1) at $S$ for any $G_{1}, G_{2}$ and $M$ such that (12) has a unique solution.

4) Matching with impulsive controllability: impulse controllability is a characteristic property of singular systems and corresponds to the controllability of only the fast subsystem. A condition for impulse controllability is given in [1]. System (1) is impulse controllable if and only if

$$
\operatorname{rank}\left[\begin{array}{ccc}
E & 0 & 0 \\
A & E & B
\end{array}\right]=n+\operatorname{rank}(E) .
$$

If the system to be reduced is impulsive controllable, it is desirable to maintain this property also in the reduced order model.

Theorem 4: System (11), or (17), or (19), is impulsive controllable if $M$ and $G_{2}$ are selected such that

$$
\operatorname{rank}\left[\begin{array}{cc}
M & G_{2}
\end{array}\right]=\operatorname{rank}(M) \text {. }
$$

The proof of the theorem is straightforward, however, to the best of the author's knowledge, the condition for impulsive controllability has not been presented in the simple form given in (21).
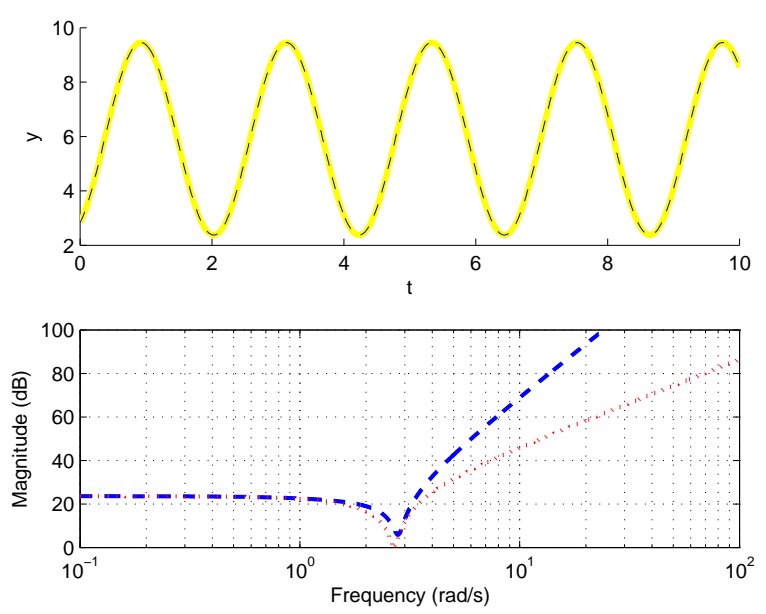

Fig. 1. (Top) Time history of the output of system (22) (solid line) and of the output of the reduced order model (23) (dashed line). (Bottom) Magnitude of the Bode plot of system (22) (dashed line) and of the reduced order model (23) (dotted line).

Proof: Following [1]

$$
\operatorname{rank}\left[\begin{array}{ccc}
E & 0 & 0 \\
A & E & B
\end{array}\right]=2 n_{1}+\operatorname{rank}\left[\begin{array}{ccc}
N & 0 & 0 \\
I & N & B_{2}
\end{array}\right] .
$$

Note now that

$$
n+\operatorname{rank}(E)=n_{1}+n_{2}+\operatorname{rank}\left[\begin{array}{cc}
I & 0 \\
0 & N
\end{array}\right] .
$$

Then condition (20) reduced to

$$
\begin{gathered}
\operatorname{rank}\left[\begin{array}{ccc}
E & 0 & 0 \\
A & E & B
\end{array}\right]=2 n_{1}+n_{2}+\operatorname{rank}\left[\begin{array}{cc}
N & B_{2}
\end{array}\right]= \\
\quad=n_{1}+n_{2}+\operatorname{rank}\left[\begin{array}{cc}
I & 0 \\
0 & N
\end{array}\right]=2 n_{1}+n_{2}+\operatorname{rank}(N),
\end{gathered}
$$

that written for the reduced order model, yields (21).

\section{EXAMPLES}

In this section we use two numerical examples to illustrate the results of the paper. The first example is a purely fast system used often in the literature of model reduction of singular systems [4], [5]. The second example is a largescale randomly generated singular system.

\section{A. A classical fast subsystem example}

This example has been proposed in [4], see also [5]. Consider the purely fast fifth order singular system described by the equations

$$
N \dot{x}=x+B u, \quad y=C x,
$$

with

$$
N=\left[\begin{array}{lllll}
0 & 1 & 0 & 0 & 0 \\
0 & 0 & 1 & 0 & 0 \\
0 & 0 & 0 & 1 & 0 \\
0 & 0 & 0 & 0 & 1 \\
0 & 0 & 0 & 0 & 0
\end{array}\right], B=\left[\begin{array}{l}
0.1 \\
0.2 \\
1.8 \\
2.5 \\
3.0
\end{array}\right], C^{\top}=\left[\begin{array}{l}
0.1 \\
0.3 \\
1.2 \\
1.8 \\
2.8
\end{array}\right]
$$



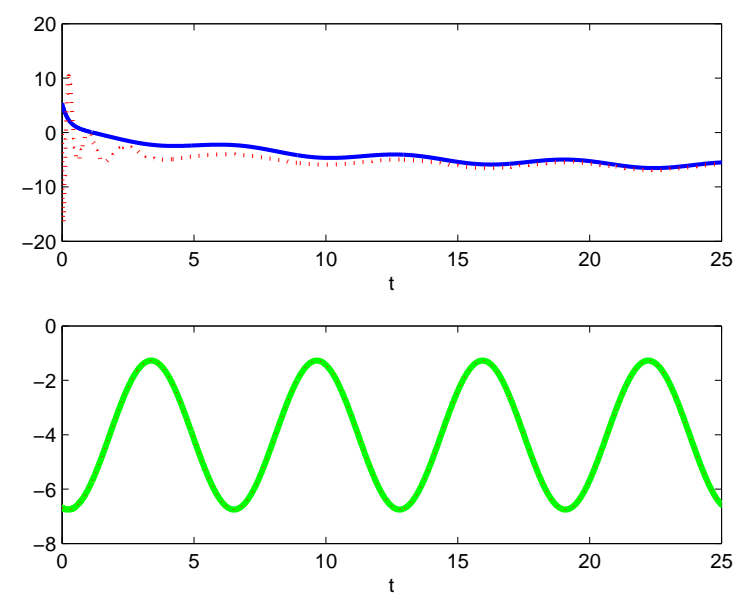

Fig. 2. (Top) Time history of the output of the slow subsystem (solid line) and the output of the slow subsystem of the reduced order model (dotted line). (Bottom) Time history of the output of the fast subsystem (solid line) and the output of the fast subsystem of the reduced order model (dotted line).

Let $\sigma(S)=\{0, \pm 2.85 j\}$ and $L$ and $\omega(0)$ in (9) be randomly generated. Then, system (17) is a third order reduced order model of system (22), namely

$$
\begin{aligned}
M \dot{\xi} & =\xi+G u, \\
\psi & =C \sum_{i=0}^{h-1} N^{i} B L S^{i}\left(\sum_{i=0}^{\hbar-1} M^{i} G L S^{i} \xi\right)^{-1} \xi,
\end{aligned}
$$

where $G$ is randomly generated and

$$
M=\left[\begin{array}{lll}
0 & 1 & 0 \\
0 & 0 & 1 \\
0 & 0 & 0
\end{array}\right] .
$$

Fig. 1 (top) shows the time history of the output of system (22) (solid line) and of the output of the reduced order model (23) (dashed line). The two responses overlap because a purely fast system does not have a transient response. Fig. 1 (bottom) shows the magnitude of the Bode plot of system (22) (dashed line) and of the reduced order model (23) (dotted line). This plot gives a more indicative information of the quality of the approximation. In fact, from the figure we see that the reduced order model is a good approximation of the system at low frequencies $(<\approx 3 \mathrm{rad} / \mathrm{s})$.

\section{B. A large-scale singular system}

Consider the singular system (2) with $n_{1}=60$ and $n_{2}=20$. Let $A_{1}=\operatorname{diag}(-0.1,-0.2, \ldots,-6.0), N$ be a canonical nilpotent matrix of degree $h=20$ and $B_{1}, B_{2}, C_{1}$, $C_{2}$ and $L$ be randomly generated. The matrix $S$ is such that $\sigma(S)=\{0, \pm 0.1 j, \pm 0.5 j, \pm j, \pm 1.13 j, \pm 5 j\}$. The reduced order model (19) is computed. The matrix $G_{1}$ is selected such that $\sigma\left(S-G_{1} L\right) \subset \mathbb{C}_{<0}, M$ is a canonical nilpotent matrix of degree $\hbar=10$ and $G_{2}$ is selected as a truncation of $B_{2}$. Fig. 2 (top) shows the time history of the output of the slow subsystem (solid line) and the output of the slow
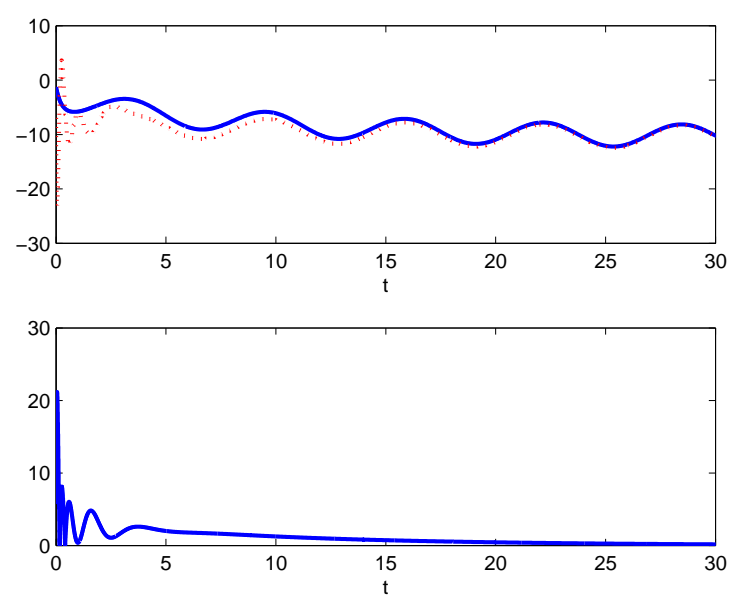

Fig. 3. (Top) Time history of the total output of the system (solid line) and of the reduced order model (dotted line). (Bottom) Absolute error between the two output responses.

subsystem of the reduced order model (dotted line). Fig. 2 (bottom) shows the time history of the output of the fast subsystem (solid line) and the output of the fast subsystem of the reduced order model (dotted line). As expected the bottom plots overlap. Fig. 3 (top) shows the time history of the total output $y$ of the system (solid line) and of the total output $\psi$ of the reduced order model (dotted line). Fig. 3 (bottom) shows the absolute error between the two output responses. As expected the error between the two responses depends only on the response of the slow subsystem and goes to zero as the transient vanishes.

\section{CONCLUSION}

The model reduction technique based on moment matching has been extended to singular systems. Combining the interpolation-based and the steady-state-based description of moment a partitioned projector is constructed. The contribution of the slow subsystem and the contribution of the fast subsystem to the moment are separated. It is clear that the information on the fast subsystem is encoded in the projector and it is not lost by the moment matching technique. Moreover, the output of reduced order models based on this projector approximates the output of the system only when consistent initial conditions are taken in account. Exploiting this partitioned projector, several families of reduced order models have been proposed. In particular, a purely fast, a purely slow and a "simple" family of reduced order models have been proposed. The possibility of maintaining the impulsive controllability property has been investigated and a few examples have been used to illustrate the results. A natural extension of the current results is represented by the model reduction of nonlinear singular system and by the model reduction of systems with constraints.

\section{REFERENCES}

[1] L. Dai, Singular Control Systems, ser. Lecture Notes in Control and Information Sciences. Springer Berlin Heidelberg, 1989. 
[2] P. Kunkel and V. L. Mehrmann, Differential-algebraic Equations: Analysis and Numerical Solution, ser. EMS textbooks in mathematics. European Mathematical Society, 2006.

[3] F. L. Lewis, M. A. Christodoulou, B. G. Mertzios, and K. Ozcaldiran, "Chained aggregation of singular systems," IEEE Transactions on Automatic Control, vol. 34, no. 9, pp. 1007-1012, Sep 1989.

[4] K. Perev and B. Shafai, "Balanced realization and model reduction of singular systems," International Journal of Systems Science, vol. 25, no. 6, pp. 1039-1052, 1994.

[5] W. Q. Liu and V. Sreeram, "Model reduction of singular systems," in Proceedings of the 39th IEEE Conference on Decision and Control, vol. 3, 2000, pp. 2373-2378.

[6] P. J. Kootsookos and R. R. Bitmead, "The Nehari shuffle and minimax FIR filter design," Control and Dynamic Systems series, vol. 64, no. 1, pp. 239-298, 1994.

[7] J. Wang, Q. Zhang, W. Liu, and V. Sreeram, "Model reduction of singular systems via covariance approximation," in Proceedings of the 2004 American Control Conference, Boston, MA, June, vol. 1, 2004, pp. 90-95.

[8] A. B. H. Adamou-Mitiche, L. Mitiche, and V. Sima, "Model reduction for descriptor systems," in First International Symposium on Control, Communications and Signal Processing, March 2004, pp. 827-830.

[9] A. Antoulas, Approximation of Large-Scale Dynamical Systems. Philadelphia, PA: SIAM Advances in Design and Control, 2005.

[10] H. Kimura, "Positive partial realization of covariance sequences," Modeling, Identification and Robust Control, pp. 499-513, 1986.

[11] C. I. Byrnes, A. Lindquist, S. V. Gusev, and A. S. Matveev, "A complete parameterization of all positive rational extensions of a covariance sequence," IEEE Transactions on Automatic Control, vol. 40, pp. 1841-1857, 1995.

[12] A. C. Antoulas, J. A. Ball, J. Kang, and J. C. Willems, "On the solution of the minimal rational interpolation problem," Linear Algebra and Its Applications, Special Issue on Matrix Problems, vol. 137-138, pp. 511-573, 1990.

[13] C. I. Byrnes, A. Lindquist, and T. T. Georgiou, "A generalized entropy criterion for Nevanlinna-Pick interpolation with degree constraint," IEEE Transactions on Automatic Control, vol. 46, pp. 822-839, 2001

[14] K. A. Gallivan, A. Vandendorpe, and P. Van Dooren, "Model reduction and the solution of Sylvester equations," in MTNS, Kyoto, 2006.

[15] C. A. Beattie and S. Gugercin, "Interpolation theory for structurepreserving model reduction," in Proceedings of the 47th IEEE Conference on Decision and Control, Cancun, Mexico, 2008.

[16] A. Astolfi, "Model reduction by moment matching for linear and nonlinear systems," IEEE Transactions on Automatic Control, vol. 55, no. 10, pp. 2321-2336, 2010.

[17] T. C. Ionescu and A. Astolfi, "Families of reduced order models that achieve nonlinear moment matching," in Proceedings of the 2013 American Control Conference, Washington, DC, USA, June 17-19, 2013, pp. 5518-5523.

[18] G. Scarciotti and A. Astolfi, "Model reduction of neutral linear and nonlinear time-invariant time-delay systems with discrete and distributed delays," Submitted to IEEE Transactions on Automatic Control, 2015.

[19] — "Model reduction for linear systems and linear time-delay systems from input/output data," in 2015 European Control Conference (to appear), Linz, July, 2015.

[20] - "Characterization of the moments of a linear system driven by explicit signal generators," in Proceedings of the 2015 American Control Conference (to appear), Chicago, IL, July, 2015.

[21] _ _ "Model reduction by matching the steady-state response of explicit signal generators," Submitted to IEEE Transactions on Automatic Control, 2015.

[22] T. C. Ionescu, A. Astolfi, and P. Colaneri, "Families of moment matching based, low order approximations for linear systems," Systems \& Control Letters, vol. 64, pp. 47-56, 2014.

[23] T. C. Ionescu and A. Astolfi, "Families of moment matching based, structure preserving approximations for linear port Hamiltonian systems," ArXiv e-prints, Apr 2013.

[24] K. Gallivan, A. Vandendorpe, and P. Van Dooren, "Sylvester equations and projection-based model reduction," Journal of Computational and Applied Mathematics, vol. 162, no. 1, pp. 213-229, 2004. 